OPEN ACCESS

Edited by:

Kai Huang,

University of Texas Medical Branch at Galveston, United States

Reviewed by:

Muhammad Usman Tariq, Abu Dhabi School of Management, United Arab Emirates Valentina Giudice,

University of Salerno, Italy

Dora Edith Corzo-Leon,

University of Aberdeen,

United Kingdom

*Correspondence:

Xiaoju LV

Ivxj33966@126.com

Specialty section: This article was submitted to Clinical Microbiology, a section of the journal Frontiers in Cellular and Infection Microbiology

Received: 04 September 2021 Accepted: 04 November 2021 Published: 22 November 2021

Citation:

Qu J, Liu X and LvX (2021)

Pulmonary Mucormycosis as

the Leading Clinical Type of Mucormycosis in Western China. Front. Cell. Infect. Microbiol. 11:770551. doi: 10.3389/fcimb.2021.770551

\section{Pulmonary Mucormycosis as the Leading Clinical Type of Mucormycosis in Western China}

\author{
Junyan $Q u^{1}$, Xijiao $L i u^{2}$ and Xiaoju $L v^{1 *}$ \\ ${ }^{1}$ Center of Infectious Disease, West China Hospital, Sichuan University, Chengdu, China, ${ }^{2}$ Radiology Department, West \\ China Hospital, Sichuan University, Chengdu, China
}

The aim was to better understand the clinical characteristics of patients with mucormycosis in western China. We retrospectively investigated the clinical, laboratory, radiological and treatment profiles of mucormycosis patients during a 10-year period (2010-2019). As a result, 59 proven mucormycosis were enrolled in this study. It was found that $52.5 \%$ of patients had worse clinical outcomes. Pulmonary mucormycosis (PM) was the most common clinical manifestation. The most frequent risk factor was diabetes mellitus $(38,64.4 \%)$ for mucormycosis patients. Cough (43, 93.5\%), fever $(24,52.2 \%)$ and hemoptysis/bloody phlegm $(21,45.7 \%)$ were the most common manifestations of PM. There were no differences in clinical manifestations, risk factors and laboratory tests between different clinical outcome groups ( $P>0.05)$. Lymph node enlargement $(30$, $65.2 \%)$, patchy shadows $(28,60.9 \%)$, cavitation $(25,53.3 \%)$ and bilateral lobe involvement $(39,84.8 \%)$ were the most common on chest CT. Nodule was more common in good outcome group $(P<0.05)$. A total of 48 cases $(81.4 \%)$ were confirmed by histopathological examination, 22 cases (37.3\%) were confirmed by direct microscopy. PM patients were treated with amphotericin B/amphotericin B liposome or posaconazale had better clinical outcomes $(P<0.05)$. In conclusion, PM was the most common clinical type of mucormycosis in China. Diabetes mellitus was the most common risk factor. PM has diverse imaging manifestations and was prone to bilateral lobes involvement. Early diagnosis and effective anti-mucor treatment contribute to successful treatment.

Keywords: mucormycosis, pulmonary mucormycosis, diabetes, clinical analysis, clinical outcome

\section{INTRODUCTION}

Mucormycosis is a rare, emerging and opportunistic fungal infection with high morbidity and mortality caused by filamentous fungi of the Mucoraceae family, order Mucorales. Mucorales fungi are ubiquitous in nature. Humans are infected mainly by inhaling sporangiospores, occasionally through the ingestion of contaminated food or traumatic inoculation (Prakash and Chakrabarti, 2019; Shariati et al., 2020). The prevalence of mucormycosis varies between developing and developed countries, ranging from 0.01 to 14 per 100000 population in Europe and India (Bitar et al., 2009; Chakrabarti and Singh, 2014; Ruhnke et al., 2015). With the growth of the number of 
immunocompromised patients, increased awareness and development of diagnostic techniques, the incidence of mucormycosis is rising (Prakash et al., 2019). According to clinical presentations, mucormycosis is mainly classified as rhino-orbito-cerebral, pulmonary, cutaneous, gastrointestinal and disseminated types (Skiada et al., 2020). The common risk factors of mucormycosis are diabetes, hematological malignancy, use of corticosteroids or immunosuppressants, and trauma (Petrikkos et al., 2014; Jeong et al., 2019). However, the main cause of mucormycosis also varies in different countries. Hematological malignancies are the main cause in countries with high income, while diabetes mellitus (DM) or trauma are the main cause in developing countries (Chakrabarti and Singh, 2014). Diagnosis of mucormycosis is challenging because of the low sensitivity and specificity of clinical diagnostic methods (Skiada et al., 2018).

The mortality of mucormycosis remains high, it may be related to delayed diagnosis, high cost of managing mucormycosis and limited treatment options (Skiada et al., 2018). Previous studies on the characteristics of mucormycosis have been conducted mainly in America, Europe and India (Prakash and Chakrabarti, 2019). Data about mucormycosis from China is sparse. The causative agents of mucormycosis vary with different geographical locations (Prakash and Chakrabarti, 2019), and the epidemiology, the clinical disease pattern of mucormycosis vary from country to country.

To better understand the clinical characteristics of patients with mucormycosis in China, in this retrospective study, we compared the demographic features, clinical presentations, laboratory data, radiographic findings and therapeutic strategies in mucormycosis patients with different clinical outcomes who were admitted to a university hospital from Jan 2010 to Dec 2019 in western China.

\section{MATERIALS AND METHODS}

\section{Patients}

From Jan 2010 to Dec 2019, the patients with a diagnosis of mucormycosis at hospital discharge were retrospectively reviewed in West China Hospital, Sichuan University, a 4,300-bed academic tertiary hospital in Chengdu, China. According to the European Organization for Research and Treatment of Cancer/Mycoses Study Group (EORTC/MSG) criteria and previous references (De Pauw et al., 2008; Petrikkos et al., 2014; Skiada et al., 2018), inclusion in the final study group required the diagnosis of proven mucormycosis as defined as follows: (1) age $\geq 14$ years; (2) clinical manifestations and radiographic findings consistent with mucormycosis; and (3) histological presence of mucormycosis in tissue specimens, and/or broad-based, ribbon-like, non-septate hyphae with right-angle branching filamentous fungi on direct microscopy of clinical specimens, and/or Mucorales species cultured from clinical specimens. Based on clinical presentation and the involvement of the body sites, rhino-orbital-cerebral, pulmonary, cutaneous and disseminated mucormycosis were classified (Jeong et al., 2019). The following data of demographic information, underlying diseases, use of corticosteroid or immunosuppressive agent, clinical manifestations, laboratory data, radiologic findings, diagnostic procedures, therapeutic strategies and clinical outcomes at 90 days were collected.

The study was approved by the Ethics Committee of West China Hospital, Sichuan University. Because all the data in this study were routinely obtained, written informed consent was waived.

\section{Laboratory Studies}

Laboratory tests including complete blood count, blood biochemistry, procalcitionin (PCT), C-reactive protein, $\mathrm{T}$ lymphocyte subset, HIV testing, serum $(1,3)$-beta-D-glucan test (BDG test), and galactomannan test (GM test) were performed. Clinical samples (including blood, sputum, secretions, urine) were aseptically collected and cultured under aerobic or anaerobic conditions. Bacterial species were isolated and identified using MicroScan WalkAway-96 System (Siemens, USA). Fungal culture was performed on Sabouraud dextrose agar (SDA) and incubated at $30^{\circ} \mathrm{C}$. All the items were performed in the Department of Laboratory Medicine of our hospital.

\section{Radiological Assessment}

Imaging examinations such as chest computed tomography (CT), abdominal CT, fibroptic bronchoscopy were performed at the discretion of the treating physicians. The CT scans were performed using 64-row multi-slice spiral CT scanner (SOMATOM definition AS+, Siemens) in our hospital. All images were reviewed independently by two experienced radiologists. For patients with $\mathrm{PM}$, chest CT findings including nodule, mass, cavity, patchy consolidation, ground glass opacity, reversed halo sign, lymph node enlargement, pleural effusion and the distribution of the lesion in the lungs were recorded.

\section{Treatment Strategies and Clinical Outcomes}

The patients with mucormycosis were treated with amphotericin B (AmB, 0.5-1 mg/kg per day) (North China Pharmaceutical Co., Ltd., China) or amphotericin B liposome (LAmB, 3-6 mg/kg per day) in accordance with the drug instructions, guidelines (Skiada et al., 2013) and patient tolerance. Posaconazole oral suspension (40mg/ml, 10ml, twice daily) (Patheon Inc, Whitby, ON, Canada) was used in patients who have contraindications to amphotericin B or who cannot tolerate the side effects of amphotericin B like reduced kidney function, electrolyte imbalances, nausea and vomiting. The treatment duration was determined by the treating physicians according to the patient's treatment response and the size of the focus. Management of patients with diabetes included dietary guidance, tight glucose control with insulin and/or hypoglycemic drugs, multiple flash glucose monitoring daily and treatment of complications. A multidisciplinary consulting team, including diabetes, infectious disease, nutrition, wound therapy, surgery, clinical microbiology, and clinical pharmacy, that offered specialist advice, on-going management. Other underlying diseases of these patients such as immune system disease, chronic lung disease were treated normally and systematically. For bacterial 
co-infection patients, empirical or targeted antimicrobial therapy were given. Clinical outcomes of patients with mucormycosis were evaluated at 90 days after diagnosis. According to clinical manifestations, laboratory findings and image changes, their clinical outcomes were divided into good and worse outcome. Death or disease progression or persistence were classified as worse outcome. The continuous improvement of clinical symptoms and imaging findings were classified as good outcome.

\section{Statistical Analysis}

Statistical analyses were performed using IBM SPSS Statistics for Windows v.26.0 (IBM Corp., Armonk, NY, USA). The ShapiroWilk normality test was used to test the normality of all quantitative variables. Continuous variables with normal distribution were presented as mean \pm standard deviation (SD) and compared by Student's t-test. The relationship between categorical variables was assessed using Chi-square test or Fisher's exact test. A two-tailed $\mathrm{P}$ value lower than 0.05 was considered statistically significant.

\section{RESULTS}

\section{Demographic Characteristics and Clinical Outcomes of Mucormycosis Patients}

A total of 68 patients with mucormycosis were admitted to our hospital from January 2010 to December 2019, 59 of whom with proven mucormycosis (mean age $54.75 \pm 14.72$ years; 44 males) were enrolled in this retrospective study. Pulmonary mucormycosis (PM) was the most commonly observed manifestation $(46 / 59,78.0 \%)$, followed by rhino-orbitalcerebral $(9 / 59,15.3 \%)$ and disseminated mucormycosis $(3 / 59$, $5.0 \%)$. There was only one case of cutaneous mucormycosis.
These patients were classified as pulmonary type and other type mucormycosis based on clinical type. The demographic and clinical outcomes of these patients were summarized in Table 1. The ages of patients with $P M$ was higher than that of patients with non-pulmonary mucormycosis $(\mathrm{P}=0.015)$. The most frequent underlying disease was diabetes mellitus (38, $64.4 \%$ ) for mucormycosis patients. More patients with PM had diabetes mellitus than patients with non-pulmonary mucormycosis $(\mathrm{P}=0.027)$. A total of 31 mucormycosis patients (52.5\%) had worse clinical outcomes, clinical outcome was not obviously different between the two groups $(\mathrm{P}>0.05)$.

\section{Demographic and Clinical Characteristics in Patients With PM}

Demographic and clinical features of PM patients with different clinical outcomes were shown in Table 2. The most common clinical symptoms of PM patients were cough (43, 93.5\%), fever $(24,52.2 \%)$ and hemoptysis/bloody phlegm $(21,45.7 \%)$. Diabetes mellitus $(33,71.7 \%)$ was the most frequent underlying disease. There were no significant difference in the gender, age, clinical symptoms and underlying diseases beween good and worse outcome group $(\mathrm{P}>0.05)$. For laboratory results, there was no significant difference between the two groups $(\mathrm{P}>0.05)$.

\section{Findings of CT Scan in Patients With PM}

The chest CT features of patients with PM were diverse, as shown in Figure 1. The detail of chest CT findings in these patients were shown in Table 3. The most common manifestations were lymph node enlargement $(30,65.2 \%)$, patchy shadows (28, $60.9 \%)$, cavitation $(25,53.3 \%)$ and nodules $(24,52.2 \%)$. Nodule was more common in good outcome group than in worse outcome group $(\mathrm{P}<0.05)$. Lymph node enlargement were more common in patients with worse outcome $(\mathrm{P}<0.05)$. Bilateral

TABLE 1 | Demographic and clinical outcomes in patients with mucormycosis.

\begin{tabular}{|c|c|c|c|c|}
\hline \multirow[t]{2}{*}{ Variables } & \multirow[b]{2}{*}{ All $(\mathbf{N}=59)$} & \multicolumn{2}{|c|}{ Clinical type } & \multirow[t]{2}{*}{ P-value } \\
\hline & & Pulmonary type $(\mathrm{N}=46)$ & Other type ( $N=13)$ & \\
\hline Male & $44(74.6)$ & $35(76.1)$ & $9(69.2)$ & 0.616 \\
\hline Age (years) (mean \pm SD) & $54.75 \pm 14.72$ & $57.41 \pm 12.40$ & $45.31 \pm 18.64$ & $0.015^{\star}$ \\
\hline \multicolumn{5}{|l|}{ Underlying diseases or risk factors } \\
\hline Diabetes mellitus & $38(64.4)$ & $33(71.7)$ & $5(38.5)$ & $0.027^{\star}$ \\
\hline Immune system disease & $2(3.4)$ & $1(2.2)$ & $1(7.7)$ & 0.395 \\
\hline Corticosteroid medication or immunosuppressive drugs & $4(6.8)$ & $3(6.5)$ & $1(7.7)$ & 1.000 \\
\hline Hematological malignancy & $3(5.1)$ & $1(2.2)$ & $2(15.4)$ & 0.119 \\
\hline Solid tumor & $2(3.4)$ & $2(4.3)$ & $0(0.0)$ & 1.000 \\
\hline Chronic kidney diseases & $3(5.1)$ & $3(6.5)$ & $0(0.0)$ & 1.000 \\
\hline Chronic lung disease & $8(13.6)$ & $7(15.2)$ & $1(7.7)$ & 0.671 \\
\hline Chronic liver disease & $2(1.7)$ & $1(2.2)$ & $1(7.7)$ & 0.395 \\
\hline Truma & $2(3.4)$ & $1(2.2)$ & $1(7.7)$ & 0.395 \\
\hline None & $8(13.6)$ & $6(13.0)$ & $2(15.4)$ & 1.000 \\
\hline \multicolumn{5}{|l|}{ Co-infection of other pathogens } \\
\hline Bacteria & $42(71.2)$ & 32 (69.6) & $10(76.9)$ & 0.738 \\
\hline Other fungi & $16(23.7)$ & $14(30.4)$ & $2(15.4)$ & 0.481 \\
\hline \multicolumn{5}{|l|}{ Clinical outcome } \\
\hline Good outcome & $28(47.5)$ & $23(50.0)$ & $5(38.5)$ & 0.462 \\
\hline Worse outcome & $31(52.5)$ & $23(50.0)$ & $8(61.5)$ & \\
\hline
\end{tabular}

${ }^{*} P<0.05$. 
TABLE 2 | Demographic and clinical characteristics in patients with pulmonary mucormycosis.

\begin{tabular}{|c|c|c|c|c|}
\hline Variables & All $(N=46)(n, \%)$ & Good outcome (N = 23) (n,\%) & Worse outcome (N = 23) (n, \%) & P-value \\
\hline Male & $35(76.1)$ & $5(21.7)$ & $6(26.1)$ & 0.288 \\
\hline Age & $57.41 \pm 12.40$ & $56.00 \pm 13.44$ & $58.82 \pm 11.38$ & 0.446 \\
\hline \multicolumn{5}{|l|}{ Presenting symptoms and signs } \\
\hline Fever & $24(52.2)$ & $13(56.5)$ & $11(47.8)$ & 0.768 \\
\hline Cough & $43(93.5)$ & $21(91.3)$ & $22(95.7)$ & 1.000 \\
\hline hemoptysis or bloody phlegm & $21(45.7)$ & $9(39.1)$ & $12(52.2)$ & 0.554 \\
\hline Chest pain & $7(15.2)$ & $2(8.7)$ & $5(21.7)$ & 0.414 \\
\hline Shortness of breath & $14(30.4)$ & $9(39.1)$ & $5(21.7)$ & 0.200 \\
\hline \multicolumn{5}{|l|}{ Underlying diseases or risk factors } \\
\hline Diabetes mellitus & $33(71.7)$ & $16(69.6)$ & $17(73.9)$ & 1.000 \\
\hline Immune system disease & $1(2.2)$ & $0(0.0)$ & $1(4.3)$ & 1.000 \\
\hline Corticosteroid medication or immunosuppressive drugs & $3(6.5)$ & $2(8.7)$ & $1(4.3)$ & 1.000 \\
\hline Hematological malignancy & $1(2.2)$ & $1(4.3)$ & $0(0.0)$ & 1.000 \\
\hline Solid tumor & $2(4.3)$ & $0(0.0)$ & $2(8.7)$ & 0.489 \\
\hline Chronic kidney diseases & $3(6.5)$ & $2(8.7)$ & $1(4.3)$ & 1.000 \\
\hline Chronic lung disease & $7(15.2)$ & $4(17.4)$ & $3(13.0)$ & 1.000 \\
\hline Chronic liver disease & $1(2.2)$ & $1(4.3)$ & $0(0.0)$ & 1.000 \\
\hline Truma & $1(2.2)$ & $1(4.3)$ & $0(0.0)$ & 1.000 \\
\hline None & $6(13.0)$ & $4(17.4)$ & $2(8.7)$ & 0.665 \\
\hline \multicolumn{5}{|l|}{ Laboratory data } \\
\hline White blood cell $\left(\times 10^{9} / \mathrm{L}\right)$ & $9.13 \pm 6.84$ & $9.58 \pm 9.02$ & $8.69 \pm 3.76$ & 0.665 \\
\hline Neutrophil (\%) & $71.96 \pm 12.24$ & $74.42 \pm 12.11$ & $69.38 \pm 12.10$ & 0.170 \\
\hline Lymphocyte (× 109/L) & $1.48 \pm 0.67$ & $1.35 \pm 0.60$ & $1.62 \pm 0.73$ & 0.180 \\
\hline Eosinophil $\left(\times 10^{9} / \mathrm{L}\right)$ & $0.33 \pm 1.17$ & $0.48 \pm 1.46$ & $0.19 \pm 0.26$ & 0.397 \\
\hline Mononuclear cell $\left(\times 10^{9} / \mathrm{L}\right)$ & $0.49 \pm 0.23$ & $0.47 \pm 0.26$ & $0.50 \pm 0.20$ & 0.539 \\
\hline Procalcitionin (ng/mL) & $0.21 \pm 0.49$ & $0.29 \pm 0.66$ & $0.12 \pm 0.13$ & 0.289 \\
\hline C-reactive protein (mg/L) & $70.31 \pm 87.94$ & $66.75 \pm 97.40$ & $73.17 \pm 82.08$ & 0.831 \\
\hline Eerythrocyte sedimentation rate $(\mathrm{mm} / \mathrm{h})$ & $55.23 \pm 24.06$ & $49.00 \pm 24.64$ & $60.42 \pm 23.31$ & 0.278 \\
\hline CD4+ T lymphocyte (\%) & $36.94 \pm 10.33$ & $37.32 \pm 8.00$ & $36.55 \pm 12.54$ & 0.771 \\
\hline CD8 $^{+}$T Iymphocyte (\%) & $31.61 \pm 9.49$ & $32.92 \pm 8.17$ & $30.30 \pm 10.80$ & 0.475 \\
\hline CD4/CD8 ratio & $1.36 \pm 0.77$ & $1.23 \pm 0.40$ & $1.48 \pm 1.01$ & 0.409 \\
\hline CD4+ T lymphocyte (cells/ $\mu$ l) & $565.45 \pm 288.69$ & $419.83 \pm 92.04$ & $740.2 \pm 357.47$ & 0.062 \\
\hline CD8 ${ }^{+}$T lymphocyte (cells/ $\mu$ l) & $396.82 \pm 176.78$ & $338.50 \pm 45.12$ & $466.80 \pm 253.69$ & 0.250 \\
\hline \multicolumn{5}{|l|}{ Co-infecting agent } \\
\hline Bacteria & $25(54.3)$ & $12(52.2)$ & $13(56.5)$ & 1.000 \\
\hline Virus & $2(4.3)$ & $1(4.3)$ & $1(4.3)$ & 1.000 \\
\hline Fungi & $18(39.1)$ & $10(43.5)$ & $8(34.8)$ & 0.763 \\
\hline
\end{tabular}

lungs were involved in $84.8 \%$ (39/46) of patients, with the left lower lobe $(20,43.5 \%)$ and right lower lobe $(18,39.1 \%)$ most commonly involved. There was no significant difference in the lobe of lesion distribution between the two groups $(\mathrm{P}>0.05)$. There were 13 patients with Mucor and Aspergillus co-infection and 1 patient with lung cancer. Based on imaging findings, 12 patients were suspected to be tumors, and 2 patients were suspected to be aspergillosis in the absence of concomitant Aspergillus. Figure 2 showed the changes of chest CT lesions during the follow-up of a patient with pulmonary fungal infection (Mucor with Aspergillus).

\section{Diagnostic Procedures and Treatment Strategies}

Of the 59 proven patients with mucormycosis, 48 cases $(81.4 \%)$ were confirmed by histopathological examination, 22 cases $(37.3 \%)$ were confirmed by direct microscopy. There was no significant difference in the diagnostic procedures between the two groups ( $\mathrm{P}>0.05)$. A total of 47 patients $(79.7 \%)$ received treatment. Treatment strategies for these patients with mucormycosis include antifungal drugs, surgery, or both. Most patients (38/59, 64.4\%) were treated with amphotericin $\mathrm{B} / \mathrm{LAmB}$. More patients with $\mathrm{PM}$ were treated with $\mathrm{AmB} / \mathrm{LAmB}(\mathrm{P}=0.006)$ or posaconazale $(\mathrm{P}=0.004)$ in good outcome group than in worse outcome group.

For patients with $\mathrm{PM}$, only 3 patients were treated with surgery and LAmB, but there was no statistical difference in clinical outcome $(\mathrm{P}>0.05)$. The most common adverse events of AmB/LAmB included renal insufficiency $(16 / 42,38.1 \%)$ and hypokalemia $(9 / 42,21.4 \%)$ in this study. The severity of all adverse events were mild to moderate (grade 1-2) according to the US National Cancer Institute Common Toxicity Criteria (NCI-CTC) (US, National Cancer Institute, 2017). The adverse reaction ratio of $\mathrm{AmB} / \mathrm{LAmB}$ has not statistical difference between the two groups $(\mathrm{P}>0.05)$. As shown in Table 4 .

\section{DISCUSSION}

Mucormycosis is rare, neglected, and associated with high mortality rates. The study found that $52.5 \%$ of patients with mucormycosis had poor clinical outcomes. Which is similar to previous research results (Jeong et al., 2019). PM was the most 

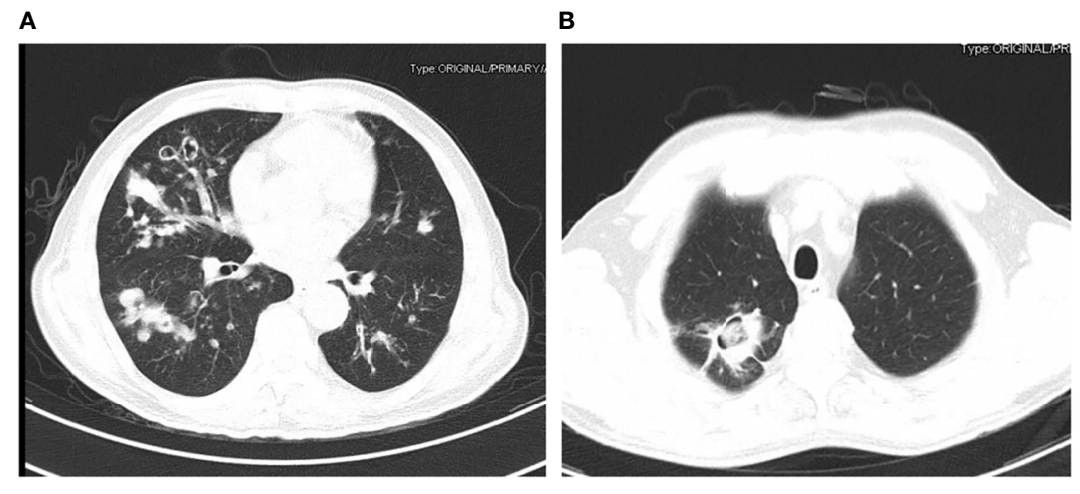

C

D
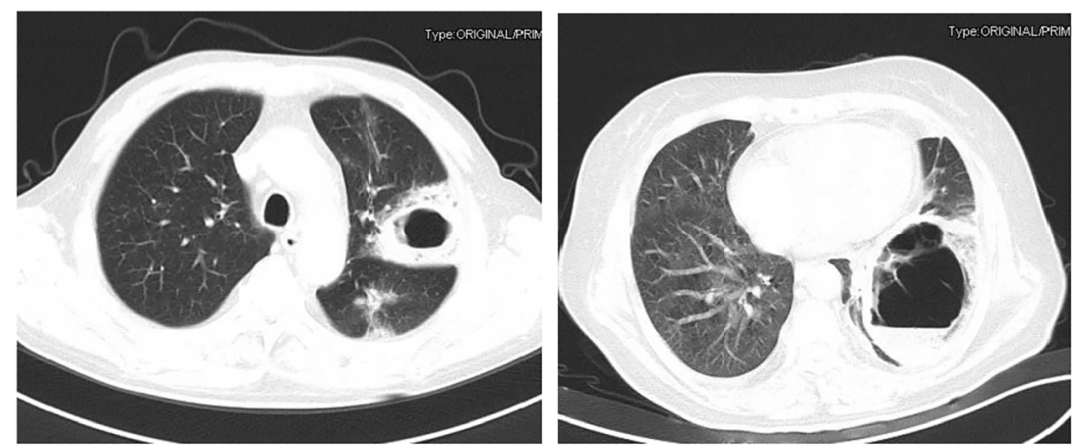

E
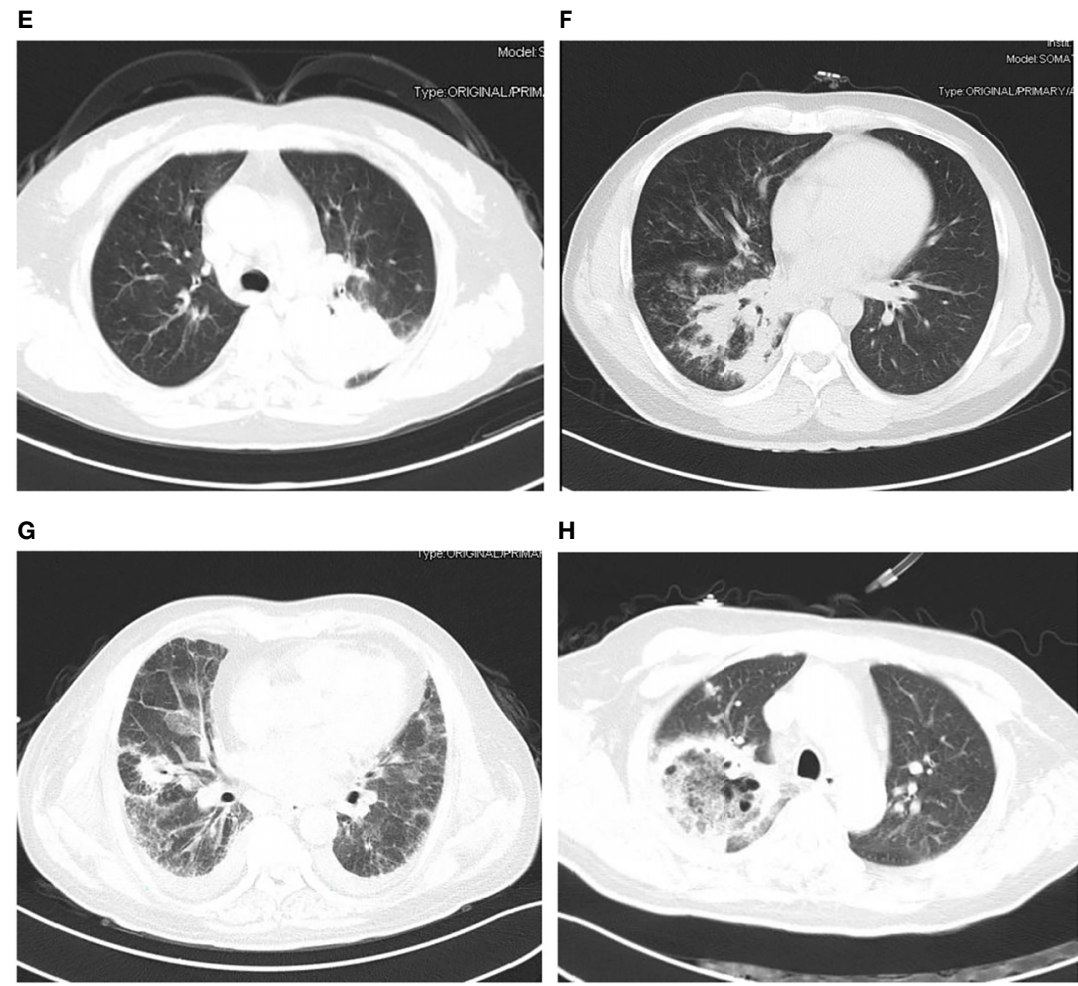

H

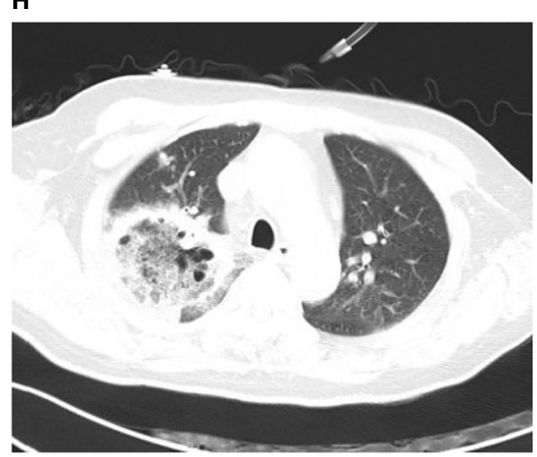

FIGURE 1 | The findings of chest computed tomography of pulmonary mucormycosis (patients with good outcome: A, B; patients with worse outcome: C-H).

(A) Multiple pleomorphic lesions in both lungs; (B) cavity with muralnodule (Mucor co-infecting with Aspergillus); (C) cavity in left lung; (D) thick-walled cavity shadow with gas-fluid level (Mucor co-infecting with Klebsiella pneumoniae); (E) mass shadow in the upper lobe of the left lung; (F) patchy shadow, nodules and consolidation in right lung; (G) cavity and ground-glass opacity; $\mathbf{( H )}$ reversed halo sign. 
TABLE 3 | Chest CT findings of proven patients with pulmonary mucormycosis .

\begin{tabular}{|c|c|c|c|c|}
\hline Morphology & All $(N=46)(n, \%)$ & Good outcome $(N=23)(n, \%)$ & Worse outcome $(N=23)(n, \%)$ & P-value \\
\hline Consolidation & $20(43.5)$ & $9(39.1)$ & $11(47.8)$ & 0.767 \\
\hline Nodule & $24(52.2)$ & $16(69.6)$ & 8 (34.8) & $0.038^{\star}$ \\
\hline Ground-glass opacity & $7(15.2)$ & $5(21.7)$ & $2(8.7)$ & 0.414 \\
\hline Mass & 15 (32.6) & 8 (34.8) & 7 (30.4) & 1.000 \\
\hline Cavitation & 25 (53.3) & $10(43.5)$ & $15(65.2)$ & 0.139 \\
\hline Patchy shadow & 28 (60.9) & $14(60.9)$ & $14(60.9)$ & 1.000 \\
\hline Fibrosis & $17(37.0)$ & 8 (34.8) & 9 (39.1) & 1.000 \\
\hline Pleural effusion & $22(47.8)$ & $11(47.8)$ & $10(43.5)$ & 1.000 \\
\hline Pleural thickening & $17(37.0)$ & $6(26.1)$ & $11(47.8)$ & 0.221 \\
\hline Lymph node enlargement & $30(65.2)$ & $11(47.8)$ & 19 (82.6) & $0.029^{*}$ \\
\hline Reversed halo sign & 5 (10.9) & $2(8.7)$ & $3(13.0)$ & 1.000 \\
\hline \multicolumn{5}{|l|}{ Lobe of lesion distribution } \\
\hline Left upper lobe & $12(26.1)$ & $5(21.7)$ & $7(30.4)$ & 0.738 \\
\hline Left lower lobe & $20(43.5)$ & $11(47.8)$ & 9 (39.1) & 0.767 \\
\hline Right upper lobe & $12(26.1)$ & $7(30.4)$ & $5(21.7)$ & 0.738 \\
\hline Right middle lobe & $11(23.9)$ & 8 (34.8) & $3(13.0)$ & 0.165 \\
\hline Right lower lobe & $18(39.1)$ & $8(34.8)$ & $10(43.5)$ & 0.763 \\
\hline Bilateral involvement & $39(84.8)$ & $20(87.0)$ & $19(82.6)$ & 1.000 \\
\hline
\end{tabular}

${ }^{*} P<0.05$.

common form of mucormycosis, accounting for $78.0 \%$ of all the patients with mucormycosis. Although previous studies have reported that rhino-orbital mucormycosis was the most common clinical type (Patel et al., 2020), PM maybe more common in China (Peng et al., 2019). This study also found the most common underlying disease was diabetes mellitus. Previous studies have shown that PM mainly occurred in patients with hematological malignancies, while rhino-orbital mucormycosis mostly occurred in patients with diabetes mellitus (Skiada et al., 2011; Chakrabarti and Singh, 2014). However, our results were consistent with those from China (Peng et al., 2019). Perhaps because the number of diabetes patients in China is much larger than that of hematological malignancies (Liu et al., 2019; Khan et al., 2020), the underlying disease of more mucormycosis patients is diabetes. The high prevalence of diabetes in China and endemic mucor species different from other countries may also be the reasons for the high incidence of PM in diabetic patients in China. More detailed etiological studies of mucormycosis need to be done. We found the incidence of mucormycosis was tended to rise, which may be owing to the improvement of fungal diagnostic technology, health awareness and the visiting rate. In addition, diabetes incidence is on the rise worldwide, especially in China and India (Khan et al., 2020), which may be the reason for the increase in the incidence of mucormycosis in China.

For PM, the most common clinical symptoms were cough, fever, and hemoptysis/bloody phlegm. This result was similar to some previous studies (Skiada et al., 2011; Peng et al., 2019). Molecular-based assays can help to identify different fungal species and it can be used as a supplement to conventional diagnostic methods (Machouart et al., 2006; Springer et al., 2016). The study also found that laboratory results were not associated with patient outcomes. Most previous studies have not found a correlation between laboratory results and clinical outcomes in patients with mucormycosis (Patel et al., 2020; Son et al., 2020), and only a few studies have found that neutropenia may be associated with increased mortality (Spellberg et al., 2012). Neutropenia was not found in our patients with PM, it might not be an important risk factor for mortality in these patients.

The most frequent imaging findings of PM were patchy shadows, cavitation and pulmonary nodules in this study. According to the imaging findings, PM might be misdiagnosed as tumor or pulmonary aspergillosis. Nodule was more common in good outcome group. Lymph node enlargement were more common in patients with worse outcome. Many previous studies have found multiple nodules, reversed halo sign, and cavities associated with PM (McAdams et al., 1997; Marom and Kontoyiannis, 2011). Pulmonary nodules, halo sign and cavitation was also found in patients pulmonary invasive aspergillosis (Althoff Souza et al., 2006; Muldoon et al., 2016). The reversed halo sign has been considered an important clue to the diagnosis of PM, however, it has also been described in other pulmonary diseases, including invasive pulmonary aspergillosis, tuberculosis, organising pneumonia and malignancy (Sullivan and Rana, 2019). Therefore, early biopsy to establish the underlying cause is very important in patients with suspected pulmonary fungal infections.

Diagnosis of mucormycosis is challenging. In clinical practice, laboratory diagnosis of mucormycosis includes histopathology, direct examination and cultures (Skiada et al., 2020). Most patients in this study were diagnosed by histopathology. Many previous studies have also reported that histopathology was the main diagnostic method of mucormycosis (Prakash and Chakrabarti, 2019). In this study, Mucorales culture positive was found only in 13 patients. It was previously reported that the positive rate of Mucorales culture could reach 79\% (Prakash and Chakrabarti, 2019). Which may be related to the low vigilance of doctors to invasive pulmonary mycosis and the absence of fungal culture for puncture specimens. More needs to be done to raise awareness of pulmonary mycosis among doctors.

In this study, twelve patients did not receive treatment and left the hospital against medical advice after diagnosis because of their critical condition and financial constraints. PM patients were treated 
A

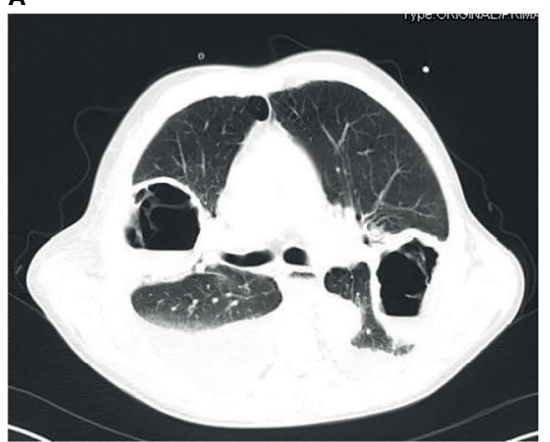

C

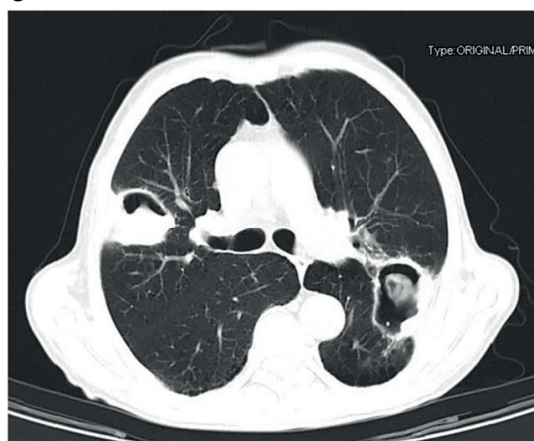

E

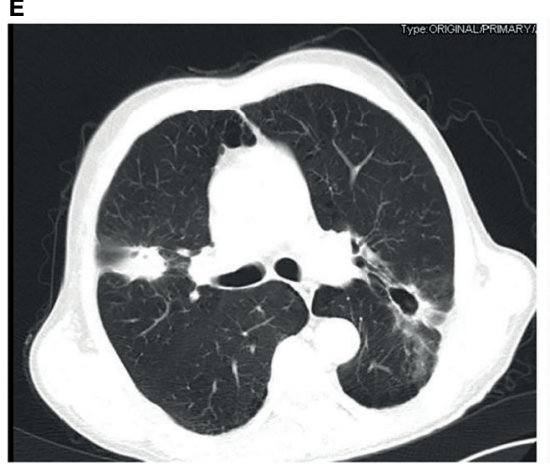

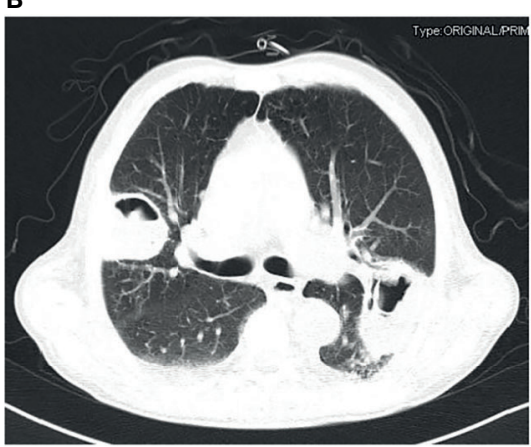

D
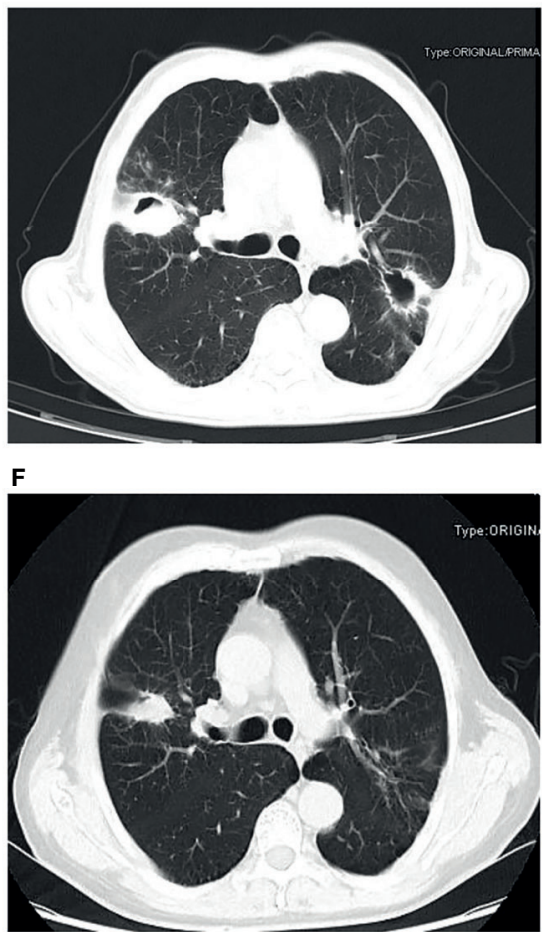

FIGURE 2 | Pulmonary fungal infection (Mucor with Aspergillus) in a 69-year-old man with uncontrolled diabetes, chronic obstructive pulmonary disease and liver cirrhosis. CT imaging showed thick-walled cavity shadow with gas-fluid level in the right upper lobe and left lower lobe of the lung (A). The lesion was significantly reduced after half a month of treatment with posaconazole (B). The lesion continued to shrink after 40 days (C), three months (D), five months (E) and 1 year (F) of treatment.

with amphotericin B or posaconazole had better clinical outcomes, while untreated patients had poor outcomes. For rhino-orbitocerebral and disseminated mucormycosis patients, there was no statistically significant difference in clinical outcome between different treatment strategies, which maybe related to the small number of cases. In addition, patients with rhino-orbito-cerebral mucormycosis who underwent surgery and antifungal therapy had better clinical outcomes. At present, treatment options for mucormycosis remain very limited. LAmB combined with surgery are strongly recommend as first-line therapy. Isavuconazole and posaconazole are also options as second-line agents (Cornely et al., 2019). For patients with PM, only 3 patients in this study were received pulmonary lobectomy, so no benefit of antifungal combined surgical treatment over antifungal therapy alone was observed. Bilateral lobe involvement occurred in $84.8 \%$ of patients in this study, which was similar to previous studies (Peng et al., 2019). Multifocal involvement limited the surgical options, early and effective antifungal therapy may be more important for patients with PM. The recommended combination of antifungal agents and surgical treatment as a treatment option for mucormycosis may be based on the presence of more rhinoorbito-cerebral mucormycosis in other countries. More researches may be needed to optimize treatment options for different types of mucormycosis. New treatment drugs or methods, such as combination of lipid amphotericin B and caspofungin or posaconazole, VT-1161, deferasirox in combination with a polyene, hyperbaric oxygen and so on, still deserve to be expected to improve clinical outcome (Skiada et al., 2018). 
TABLE 4 | Diagnostic procedures and treatment strategies for proven patients with mucormycosis .

\begin{tabular}{|c|c|c|c|c|}
\hline Diagnostic method & All $(N=59)(n, \%)$ & Good outcome (N = 28) (n,\%) & Worse outcome $(N=31)(n, \%)$ & P-value \\
\hline Direct microscopy & $22(37.3)$ & $7(25.0)$ & $15(48.4)$ & 0.105 \\
\hline Histology & $48(81.4)$ & $23(82.1)$ & $25(80.6)$ & 1.000 \\
\hline Direct microscopy positive only & $2(3.4)$ & $1(3.6)$ & $1(3.2)$ & 1.000 \\
\hline Histology positive only & $25(52.4)$ & $14(50.1)$ & $11(35.5)$ & 0.260 \\
\hline Cluture+histology & $4(6.8)$ & $2(7.1)$ & $2(6.5)$ & 1.000 \\
\hline BDG test & $3(5.1)$ & $1 / 24(4.2)$ & 2/25 (8.0) & 1.000 \\
\hline GM test & $3(5.1)$ & 2/20 (10.0) & $1 / 20(5.0)$ & 1.000 \\
\hline \multicolumn{5}{|l|}{ Therapeutic strategies } \\
\hline Pulmonary & $\begin{array}{c}\text { All } \\
(\mathrm{N}=46)(\mathrm{n}, \%)\end{array}$ & $\begin{array}{l}\text { Good outcome } \\
(N=23)(n, \%)\end{array}$ & $\begin{array}{l}\text { Worse outcome } \\
(N=23)(n, \%)\end{array}$ & P-value \\
\hline AmB/LAmB & $28(60.9)$ & $19(82.6)$ & $9(39.1)$ & $0.006^{\star}$ \\
\hline AmB/LAmB & $7(77.8)$ & $2(66.7)$ & $5(83.3)$ & 1.000 \\
\hline Posaconazale & $1(11.1)$ & $0(0.0)$ & $1(16.7)$ & 1.000 \\
\hline AmB+Surgery & $1(22.2)$ & $1(33.3)$ & $0(0.0)$ & 0.333 \\
\hline Disseminated & $\begin{array}{c}\text { All } \\
(N=3)(n, \%)\end{array}$ & $\begin{array}{l}\text { Good outcome } \\
\qquad(N=1)(n, \%)\end{array}$ & $\begin{array}{l}\text { Worse outcome } \\
\qquad(\mathrm{N}=2)(\mathrm{n}, \%)\end{array}$ & P-value \\
\hline AmB & $2(66.7)$ & $1(100.0)$ & $1(50.0)$ & NA \\
\hline untreated & $1(33.3)$ & $0(0.0)$ & $1(50.0)$ & NA \\
\hline Cutaneous & $\begin{array}{c}\text { All } \\
(N=1)(n, \%)\end{array}$ & $\begin{array}{l}\text { Good outcome } \\
\qquad(N=1)(n, \%)\end{array}$ & $\begin{array}{l}\text { Worse outcome } \\
\qquad(\mathrm{N}=0)(\mathrm{n}, \%)\end{array}$ & P-value \\
\hline AmB+Surgery & $1(100.0)$ & $0(100.0)$ & $0(0.0)$ & NA \\
\hline \multicolumn{5}{|l|}{ Adverse reactions of AmB/LAmB } \\
\hline Pulmonary & $\begin{array}{c}\text { All } \\
(\mathrm{N}=31)(\mathrm{n}, \%)\end{array}$ & $\begin{array}{l}\text { Good outcome } \\
(N=20)(n, \%)\end{array}$ & $\begin{array}{l}\text { Worse outcome } \\
\qquad(N=11)(n, \%)\end{array}$ & P-value \\
\hline Hypokalemia & $1(1.3)$ & $0(0.0)$ & $1(20.0)$ & NA \\
\hline Renal insufficiency & $4(50.0)$ & $3(100.0)$ & $1(20.0)$ & NA \\
\hline Disseminated & $\begin{array}{c}\text { All } \\
(\mathrm{N}=2)(\mathrm{n}, \%)\end{array}$ & $\begin{array}{l}\text { Good outcome } \\
\qquad(N=1)(n, \%)\end{array}$ & $\begin{array}{l}\text { Worse outcome } \\
\qquad(N=1)(n, \%)\end{array}$ & P-value \\
\hline Hypokalemia & $1(50.0)$ & $0(0.0)$ & $1(100.0)$ & NA \\
\hline Renal insufficiency & $1(50.0)$ & $1(100.0)$ & $0(0.0)$ & NA \\
\hline Gastrointestinal toxicity & $1(50.0)$ & $0(0.0)$ & $1(100.0)$ & NA \\
\hline Agranulocytosis & $1(50.0)$ & $0(0.0)$ & $1(100.0)$ & NA \\
\hline
\end{tabular}

AmB, amphotericin B; BDG test, (1,3)-beta-D-glucan test; GM test, galactomannan test; LAmB, liposomal amphotericin B.

${ }^{*} P<0.05 ;$ NA, Not Applicable.

This study has some limitations. First, it was a retrospective observational study. Only some patients have complete followup data in our hospital, especially imaging data, and the followup of some patients could only be completed by telephone. Second, the detection rate of tissue culture was low, the pathological diagnosis was determined mainly by morphology and special staining. It is important to raise the awareness of doctors about fungal disease and the importance of tissue culture. Third, because mucormycosis is a relatively rare fungal disease in China (Liao et al., 2013), the number of cases in this study was limited. More larger-scale, multicenter studies of mucormycosis in the real world should be done.

\section{CONCLUSIONS}

The most common clinical type of mucormycosis in China was PM. The most common risk factor was diabetes mellitus. Diabetic patients with clinical manifestations of febrile and 
hemoptysis, CT findings of nodules, cavities and bilateral lung involvement should be vigilant against PM. Early diagnosis and effective anti-mucor treatment are very important to improve the prognosis of patients with mucormycosis.

\section{DATA AVAILABILITY STATEMENT}

The original contributions presented in the study are included in the article. Further inquiries can be directed to the corresponding author.

\section{ETHICS STATEMENT}

The studies involving human participants were reviewed and approved by Ethics Committee of West China Hospital, Sichuan University. Written informed consent for participation was not

\section{REFERENCES}

Althoff Souza, C., Muller, N. L., Marchiori, E., Escuissato, D. L., and Franquet, T. (2006). Pulmonary Invasive Aspergillosis and Candidiasis in Immunocompromised Patients: A Comparative Study of the HighResolution CT Findings. J. Thorac. Imaging 21 (3), 184-189. doi: 10.1097/ 01.rti.0000213552.16011.ad

Bitar, D., Van Cauteren, D., Lanternier, F., Dannaoui, E., Che, D., Dromer, F., et al. (2009). Increasing Incidence of Zygomycosis (Mucormycosis), France, 19972006. Emerg. Infect. Dis. 15 (9), 1395-1401. doi: 10.3201/eid1509.090334

Chakrabarti, A., and Singh, R. (2014). Mucormycosis in India: Unique Features. Mycoses 57 (Suppl 3), 85-90. doi: 10.1111/myc.12243

Cornely, O. A., Alastruey-Izquierdo, A., Arenz, D., Chen, S. C. A., Dannaoui, E., Hochhegger, B., et al. (2019). Global Guideline for the Diagnosis and Management of Mucormycosis: An Initiative of the European Confederation of Medical Mycology in Cooperation With the Mycoses Study Group Education and Research Consortium. Lancet Infect. Dis. 19 (12), e405-e421. doi: 10.1016/S1473-3099(19)30312-3

De Pauw, B., Walsh, T. J., Donnelly, J. P., Stevens, D. A., Edwards, J. E., Calandra, T., et al. (2008). Revised Definitions of Invasive Fungal Disease From the European Organization for Research and Treatment of Cancer/Invasive Fungal Infections Cooperative Group and the National Institute of Allergy and Infectious Diseases Mycoses Study Group (EORTC/MSG) Consensus Group. Clin. Infect. Dis. 46 (12), 1813-1821. doi: 10.1086/588660

Jeong, W., Keighley, C., Wolfe, R., Lee, W. L., Slavin, M. A., Kong, D. C. M., et al. (2019). The Epidemiology and Clinical Manifestations of Mucormycosis: A Systematic Review and Meta-Analysis of Case Reports. Clin. Microbiol. Infect. 25 (1), 26-34. doi: 10.1016/j.cmi.2018.07.011

Khan, M. A. B., Hashim, M. J., King, J. K., Govender, R. D., Mustafa, H., and Al Kaabi, J. (2020). Epidemiology of Type 2 Diabetes - Global Burden of Disease and Forecasted Trends. J. Epidemiol. Glob Health 10 (1), 107-111. doi: 10.2991/ jegh.k.191028.001

Liao, Y., Chen, M., Hartmann, T., Yang, R. Y., and Liao, W. Q. (2013). Epidemiology of Opportunistic Invasive Fungal Infections in China: Review of Literature. Chin. Med. J. (Engl) 126 (2), 361-368.

Liu, W., Liu, J., Song, Y., Zeng, X., Wang, X., Mi, L., et al. (2019). Burden of Lymphoma in China, 2006-2016: An Analysis of the Global Burden of Disease Study 2016. J. Hematol. Oncol. 12 (1), 115. doi: 10.1186/s13045-019-0785-7

Machouart, M., Larché, J., Burton, K., Collomb, J., Maurer, P., Cintrat, A., et al. (2006). Genetic Identification of the Main Opportunistic Mucorales by PCRRestriction Fragment Length Polymorphism. J. Clin. Microbiol. 44, 805-810. doi: 10.1128/JCM.44.3.805-810.2006 required for this study in accordance with the national legislation and the institutional requirements.

\section{AUTHOR CONTRIBUTIONS}

XL and JQ conceived of and designed the study. JQ and XL collected, analysed or interpreted data. JQ wrote the draft. All authors read, revised and approved the final manuscript.

\section{FUNDING}

This study was supported by Sichuan Province Science and Technology Support Program of China (grant number: 2021YFS0170), $1 \cdot 3 \cdot 5$ project for disciplines of excellenceClinical Research Incubation Project, West China Hospital, Sichuan University (grant number: 2021HXFH032).

Marom, E. M., and Kontoyiannis, D. P. (2011). Imaging Studies for Diagnosing Invasive Fungal Pneumonia in Immunocompromised Patients. Curr. Opin. Infect. Dis. 24 (4), 309-314. doi: 10.1097/QCO.0b013e328348b2e1

McAdams, H. P., Rosado de Christenson, M., Strollo, D. C., and Patz, E. F. Jr. (1997). Pulmonary Mucormycosis: Radiologic Findings in 32 Cases. AJR Am. J. Roentgenol 168 (6), 1541-1548. doi: 10.2214/ajr.168.6.9168721

Muldoon, E. G., Sharman, A., Page, I., Bishop, P., and Denning, D. W. (2016). Aspergillus Nodules; Another Presentation of Chronic Pulmonary Aspergillosis. BMC Pulm Med. 16 (1), 123. doi: 10.1186/s12890-0160276-3

Patel, A., Kaur, H., Xess, I., Michael, J. S., Savio, J., Rudramurthy, S., et al. (2020). A Multicentre Observational Study on the Epidemiology, Risk Factors, Management and Outcomes of Mucormycosis in India. Clin. Microbiol. Infect. 26 (7), 944 e949-944.e915. doi: 10.1016/j.cmi.2019.11.021

Peng, M., Meng, H., Sun, Y., Xiao, Y., Zhang, H., Lv, K., et al. (2019). Clinical Features of Pulmonary Mucormycosis in Patients With Different Immune Status. J. Thorac. Dis. 11 (12), 5042-5052. doi: 10.21037/jtd.2019.12.53

Petrikkos, G., Skiada, A., and Drogari-Apiranthitou, M. (2014). Epidemiology of Mucormycosis in Europe. Clin. Microbiol. Infect. 20 (Suppl 6), 67-73. doi: 10.1111/1469-0691.12563

Prakash, H., and Chakrabarti, A. (2019). Global Epidemiology of Mucormycosis. J. Fungi (Basel) 5 (1), 26. doi: 10.3390/jof5010026

Prakash, H., Ghosh, A. K., Rudramurthy, S. M., Singh, P., Xess, I., Savio, J., et al. (2019). A Prospective Multicenter Study on Mucormycosis in India: Epidemiology, Diagnosis, and Treatment. Med. Mycol 57 (4), 395-402. doi: 10.1093/mmy/myy060

Ruhnke, M., Groll, A. H., Mayser, P., Ullmann, A. J., Mendling, W., Hof, H., et al. (2015). University of Manchester in Association With the Lp: Estimated Burden of Fungal Infections in Germany. Mycoses 58 (Suppl 5), 22-28. doi: 10.1111/ myc. 12392

Shariati, A., Moradabadi, A., Chegini, Z., Khoshbayan, A., and Didehdar, M. (2020). An Overview of the Management of the Most Important Invasive Fungal Infections in Patients With Blood Malignancies. Infect. Drug Resist. 13, 2329-2354. doi: 10.2147/IDR.S254478

Skiada, A., Lanternier, F., Groll, A. H., Pagano, L., Zimmerli, S., Herbrecht, R., et al. (2013). Diagnosis and Treatment of Mucormycosis in Patients With Hematological Malignancies: Guidelines From the 3rd European Conference on Infections in Leukemia (ECIL 3). Haematologica 98 (4), 492-504. doi: 10.3324/haematol.2012.065110

Skiada, A., Lass-Floerl, C., Klimko, N., Ibrahim, A., Roilides, E., and Petrikkos, G. (2018). Challenges in the Diagnosis and Treatment of Mucormycosis. Med. Mycol 56 (suppl_1), 93-101. doi: 10.1093/mmy/myx101 
Skiada, A., Pagano, L., Groll, A., Zimmerli, S., Dupont, B., Lagrou, K., et al. (2011). Gaustad P Et Al: Zygomycosis in Europe: Analysis of 230 Cases Accrued by the Registry of the European Confederation of Medical Mycology (ECMM) Working Group on Zygomycosis Between 2005 and 2007. Clin. Microbiol. Infect. 17 (12), 1859-1867. doi: 10.1111/j.1469-0691.2010.03456.x

Skiada, A., Pavleas, I., and Drogari-Apiranthitou, M. (2020). Epidemiology and Diagnosis of Mucormycosis: An Update. J. Fungi (Basel) 6 (4), 265. doi: 10.3390/jof6040265

Son, H. J., Song, J. S., Choi, S., Jung, J., Kim, M. J., Chong, Y. P., et al. (2020). Risk Factors for Mortality in Patients With Pulmonary Mucormycosis. Mycoses 63 (7), 729-736. doi: 10.1111/myc.13092

Spellberg, B., Kontoyiannis, D. P., Fredricks, D., Morris, M. I., Perfect, J. R., ChinHong, P. V., et al. (2012). Risk Factors for Mortality in Patients With Mucormycosis. Med. Mycol 50 (6), 611-618. doi: 10.3109/13693786.2012. 669502

Springer, J., Lackner, M., Ensinger, C., Risslegger, B., Morton, CO., Nachbaur, D., et al. (2016). Clinical Evaluation of a Mucorales-Specific Real-Time PCR Assay in Tissue and Serum Samples. J. Med. Microbiol. 65, 1414-1421. doi: 10.1099/ jmm.0.000375

Sullivan, T., and Rana, M. (2019). The Reversed Halo Sign and Mucormycosis. Lancet Infect. Dis. 19 (12), 1379. doi: 10.1016/S1473-3099(19)30548-1
US, National Cancer Institute (2017). Common Terminology Criteria for Adverse Events (CTCAE): Version 5.0. Available from: https://ctep.cancer.gov/ protocoldevelopment/electronic_applications/docs/CTCAE_v5_Quick_ Reference_8.5x11.pdf. (Accessed December13, 2021).

Conflict of Interest: The authors declare that the research was conducted in the absence of any commercial or financial relationships that could be construed as a potential conflict of interest.

Publisher's Note: All claims expressed in this article are solely those of the authors and do not necessarily represent those of their affiliated organizations, or those of the publisher, the editors and the reviewers. Any product that may be evaluated in this article, or claim that may be made by its manufacturer, is not guaranteed or endorsed by the publisher.

Copyright $(2) 2021 \mathrm{Qu}$, Liu and Lv. This is an open-access article distributed under the terms of the Creative Commons Attribution License (CC BY). The use, distribution or reproduction in other forums is permitted, provided the original author(s) and the copyright owner(s) are credited and that the original publication in this journal is cited, in accordance with accepted academic practice. No use, distribution or reproduction is permitted which does not comply with these terms. 